\title{
MESHLESS TECHNIQUES FOR EFFICIENT SIMULATION OF NON-LINEAR BEHAVIOR IN ELECTROSTATIC MEMS
}

\author{
Gang Li and N. R. Aluru* \\ Beckman Institute for Advanced Science and Technology \\ University of Illinois at Urbana-Champaign \\ Urbana, IL 61801
}

\begin{abstract}
Accurate analysis of electrostatic MEMS and the development of reliable reduced-order (or macro) models for rapid system-level analysis requires proper understanding and the characterization of the non-linearity in electrostatic devices. The use of linear theories instead of non-linear theories can produce inaccurate results and the use of non-linear theories, in situations where linear theories can produce accurate results, can be very expensive and time consuming. Hence, it is critical to identify the regimes where linear and non-linear theories are valid. Simulation of non-linear behavior in electrostatic devices using classical finite-difference and finite-element methods can be very inefficient because of complex meshing requirements. We propose new nonlinear meshless techniques for efficient simulation of electrostatic MEMS. The key results of this paper are two-fold: (i) the development of new meshless techniques for radically simpler analysis of electrostatic MEMS and (ii) the identification of the regimes where linear and non-linear theories are accurate and the various parameters that contribute to the non-linearity in electrostatic devices.
\end{abstract}

\section{INTRODUCTION}

Meshless techniques [1-3] are extremely powcrful numerical methods as they do not require the generation of a mesh for complex three-dimensional microelectromechanical devices and systems (MEMS). Meshless techniques radically simplify the computer-aided design (CAD) for MEMS and significantly reduce the design cycle time for innovative MEMS. In this paper, we present a new finite cloud meshless method for geometrically nonlinear analysis of electrostatic devices. The key features of the method are (i) it is a true meshless technique - which means that only points need to be sprinkled and no connectivity information among the points is necessary (ii) a fixed reproducing kernel approximation is employed for constructing the interpolation functions and (iii) a collocation method is used for discretizing the governing equations.

By using the finite-cloud meshless method we investigate the regimes where linear and non-linear theories are suitable for electrostatic MEMS. The peak deflection and pull-in voltage obtained with linear and non-linear theories for a cantilever beam and a fixed-fixed beam with various lengths and gaps are studied. It is shown that for a small gap, both linear and non-linear theories give identical results, which are in excellent agreement with published data for pull-in voltages [5]. However, as the gap/length ratio increases, with all other variables held fixed, significant

\footnotetext{
* This research is supported by DARPA under agreement number F30602-98-2-0718. Address all correspondence to aluru@ uiuc.edu http://www. staff.uiuc.edu/ aluru/.
}

deviations are observed between linear and nonlinear theories. Specifically, we have observed that, for larger gaps in comparison with the length, the pull-in voltages obtained with non-linear theories are accurate, and the linear theories are in significant error. The comparison between linear and non-linear theories for the fixed-fixed beam is much more pronounced. As the gap to length ratio increases, the error in pull-in voltage with linear theory increases significantly. From the results presented in this paper, it is evident that the gap/length ratio is one of the key variables in determining whether a linear or a non-linear theory should be employed for electrostatic MEMS design.

\section{GOVERNING EQUATIONS}

We will be primarily concerned with two-dimensional devices in this paper. For small micro-mechanical deformations, the linear theory of elasticity as defined by equations (1)-(3) can be used.

$$
\begin{array}{cc}
\nabla \cdot \tau+b=0 & \text { in } \Omega \\
\boldsymbol{u}=\boldsymbol{g} & \text { on } \Gamma_{g} \\
\tau \cdot \boldsymbol{n}=\boldsymbol{h} & \text { on } \Gamma_{h}
\end{array}
$$

In the above equations, $\tau$ is the Cauchy stress, $b$ is the body force, $\boldsymbol{g}$ is the specified displacement on the boundary portion $\Gamma_{\mathrm{g}}$ and $\boldsymbol{h}$ is the surface traction on the boundary $\Gamma_{h}, n$ is the unit outward normal. In coupled electro-mechanical analysis, the surface tractions are nothing but the electrostatic forces. When the electrostatic forces are dominant, the micro-mechanical structure can undergo large deformations, in which case the linear theory described by equations (1)-(3) may produce inaccurate results.

When an elastic body undergoes large deformation, the difference between the initial configuration and the deformed configuration of the body can not be neglected as was done for the case of linear elasticity. Instead, Eq. (1) is only valid for the deformed body whose configuration is unknown. While Cauchy stress and engineering strain are used as stress and strain measures for linear theory, the second Piola-Kirchhoff stress and the GreenLagrangian strain are used as the stress and strain measures, respectively, for the geometrically non-linear analysis. The governing equations for large deformation analysis can be described by using either a total Lagrangian or an updated Lagrangian description (see [3] for differences between the two descriptions). In this paper we use a total Lagrangian description, in which all the variables reside in the initial configuration. The governing equations for a large deformation case are summarized below:

$$
\begin{array}{cc}
\nabla \cdot(\boldsymbol{F S})+\boldsymbol{B}=0 & \text { in } \Omega \\
\boldsymbol{u}=\boldsymbol{G} & \text { on } \Gamma_{g} \\
\boldsymbol{P} \cdot \boldsymbol{N}=\boldsymbol{H} & \text { on } \Gamma_{h}
\end{array}
$$

where $\boldsymbol{F}$ is the deformation gradient

$$
F=I+\nabla u
$$


$\boldsymbol{u}$ is the displacement from the initial configuration $X$ to the deformed configuration $x, x=X+u, S$ is the second PiolaKirchhoff stress given by:

$$
S=C E
$$

where $C$ is the material tensor and $E$ is the Green-Lagrangian strain:

$$
E=\frac{1}{2}\left(F^{T} F-I\right)
$$

$\boldsymbol{B}$ is the body force per unit undeformed volume and $\boldsymbol{H}$ is the surface traction per unit undeformed area. $\boldsymbol{P}$ is the first PiolaKirchhoff stress tensor given by

$$
P=F S
$$

\section{FINITE CLOUD MESHLESS METHOD}

The finite cloud meshless (FCM) method uses a fixed reproducing kernel approximation to construct the interpolation functions and a point collocation technique for discretizing the governing partial differential equations [1]. In a fixed reproducing kernel technique, an approximation $u^{a}(x, y)$ to an unknown $u(x, y)$ is given by

$$
u^{a}(x, y)=\int_{\Omega} C(x, y, s, t) \varphi\left(x_{K}-s, y_{K}-t\right) u(s, t) d s d t
$$

Where the correction function $C(x, y, s, t)$ is given by

$$
C(x, y, s, t)=\boldsymbol{P}^{T}(s, t) C(x, y)
$$

$\varphi$ is the kernel function which is usually taken as a cubic spline or a Gaussian function. $\boldsymbol{P}$ is the vector of basis functions

$$
\boldsymbol{P}^{T}= \begin{cases}{[1, s, t]} & m=3 \\ {\left[1, s, t, s^{2}, s t, t^{2}\right]} & m=6\end{cases}
$$

$C(x, y)$ are the unknown correction function coefficients computed by satisfying the reproducing conditions, i.e.

$$
\begin{array}{r}
\int_{\Omega} \boldsymbol{P}^{T}(s, t) \boldsymbol{C}(x, y) \varphi\left(x_{K}-s, y_{k}-t\right) p_{i}(s, t) d s d t=p_{i}(x, y) \\
i=1,2, \ldots, m
\end{array}
$$

In discrete form, Eq. (14) can be written as

$$
\begin{array}{r}
\sum_{I=1}^{N P} P^{T}\left(x_{I}, y_{I}\right) C(x, y) \varphi\left(x_{K}-x_{I}, y_{K}-y_{I}\right) p_{i}\left(x_{I}, y_{I}\right) \Delta V_{I}=p_{i}(x, y) \\
i=1,2, \ldots, m
\end{array}
$$

Eq. (15) can be rewritten in a matrix form as

$$
\begin{array}{r}
M C(x, y)=P(x, y) \\
M_{i j}=\sum_{l=1}^{N P} p_{i}\left(x_{l}, y_{I}\right) \varphi\left(x_{K}-x_{I}, y_{K}-y_{l}\right) p_{j}\left(x_{I}, y_{I}\right) \Delta V_{I} \\
i, j=1,2, \ldots, m
\end{array}
$$

From Eq. (16), the unknown correction function coefficients are computed as

$$
C(x, y)=M^{-1} P(x, y)
$$

Substituting the correction function coefficients into Eq. (11) and employing a discrete approximation for Eq. (11), we obtain

$$
u^{a}(x, y)=\sum_{l=1}^{N P} N_{I}(x, y) \hat{u}_{I}
$$

where $\hat{u}_{l}$ is the nodal parameter for node $I$, and $N_{l}(x, y)$ is the fixed reproducing kernel interpolation function defined as $N_{I}(x, y)=\boldsymbol{P}^{T}(x, y) \boldsymbol{M}^{-1} \boldsymbol{P}\left(x_{I}, y_{I}\right) \varphi\left(x_{K}-x_{I}, y_{K}-y_{I}\right) \Delta V_{I}$

The derivatives of the unknown $u$ are approximated by

$$
\frac{\partial u^{a}}{\partial x}=\sum_{i=1}^{N R} \frac{\partial N_{I}(x, y)}{\partial x} \hat{u}_{I}
$$

$$
\frac{\partial^{2} u^{a}}{\partial x^{2}}=\sum_{I=1}^{N P} \frac{\partial^{2} N_{I}(x, y)}{\partial x^{2}} \hat{u}_{I}
$$

\section{FCM FOR LARGE DEFORMATION ANALYSIS}

For large deformation analysis the displacements $\boldsymbol{u}, \boldsymbol{v}$ and their derivatives are approximated by using Eq. (20)-(22). Substituting the approximations into Eq. (4), one gets a non-linear system of equations

$$
f_{j}\left(\hat{u}_{1}, \hat{u}_{2}, \ldots, \hat{u}_{N P}, \hat{v}_{1}, \hat{v}_{2}, \ldots, \hat{v}_{N P}\right)=0 \quad j=1,2
$$

This non-linear system can be solved by using a Newton's method. Boundary conditions are enforced on the boundary points where Dirichlet or Neumann boundary conditions are specified. The governing equations for such boundary points are

$$
\begin{array}{ll}
\boldsymbol{u}-\boldsymbol{G}=0 & \text { on } \Gamma_{g} \\
\boldsymbol{P} \cdot \boldsymbol{N}-\boldsymbol{H}=0 & \text { on } \Gamma_{h}
\end{array}
$$

The accuracy of FCM is demonstrated by computing the large deformation of a cantilever beam. In Figure 1, we consider the large deformation of a $10 \mu \mathrm{m} \times 1 \mu \mathrm{m} \times 1 \mu \mathrm{m}$ cantilever beam. In Figure 2, the results obtained with the meshless method are compared with ANSYS results. The good match between the two results establishes the accuracy of the non-linear meshless technique. In Figure 3, we simulate the same beam by using a random distribution of 455 points. A comparison with the analytical solution is shown in Figure 4. This result clearly establishes the significance of the method - by simply sprinkling points randomly, we can obtain accurate results without having to go through the complex process of generating meshes for micromachined devices.

\section{MESHLESS ANALYSIS OF ELECTROSTATIC MEMS}

The behavior of many microswitches can be characterized by studying the deformation of cantilever and fixed-fixed beams subjected to electrostatic potentials. The linear and non-linear models presented in Eq. (1)-(3) and Eq. (4)-(6), respectively, are solved when the surface tractions are the electrostatic forces.

In Figures 5-10, we investigate the regimes where linear and non-linear theories are appropriate. Cantilever and fixed-fixed beams with a width of $10 \mu \mathrm{m}$ and a thickness of $0.5 \mu \mathrm{m}$ are considered. Figure 5 and 6 compare the peak deflection and pull-in voltage obtained with linear and non-linear theories for a fixedfixed beam with a length of $70 \mu \mathrm{m}$ and two gap lengths. For a small gap length of $0.5 \mu \mathrm{m}$ (shown in Figure 5), we observe that both linear and non-lincar thcorics give identical results. However, for a large gap length of $2 \mu \mathrm{m}$ (shown in Figure 6), we observe that the pull-in voltage is significantly different. More extensive studies for the cantilever beam with lengths varying from $10 \mu \mathrm{m}$ to $100 \mu \mathrm{m}$ and gap/length ratios varying from $1 \%$ to $60 \%$ are shown in Figures 7 and 8. For small gaps in comparison with the length of the beam, we observe that the pull-in voltages obtained with both linear and non-linear theories are very close. However, for larger gaps in comparison with the length, we observe that the pull-in voliages obtained with linear theories are in significant error compared to non-linear theories. The comparison between linear and non-linear theories for the fixed-fixed beam, shown in Figures 9 and 10, is more pronounced. As the gap to length ratio increases, the error in pull-in voltage with the linear theory 
increases significantly. Furthermore, for the fixed-fixed beam example, for a fixed gap/length ratio, the change of length has a significant effect on the pull-in voltage error while this effect is not very pronounced in the cantilever beam case.

\section{CONCLUSIONS}

The primary contributions of the paper are summarized as follows: (a) For electrostatic structures, linear theories hold over a very small regime (gap/length ratio of less than $10 \%$ for cantilever beams and less than $1 \%$ for fixed-fixed beams). For accurate simulations or for the development of reduced-order models, designers need to use non-linear theories appropriately (b) We have developed new non-linear meshless techniques to efficiently and accurately simulate the non-linear behavior of electrostatic devices. These approaches offer radically simpler and easy-to-use CAD tools for MEMS designers.

\section{REFERENCES}

1. N. R. Aluru and Gang Li, "Finite Cloud Method: A True Meshless Technique Based On A Fixed Reproducing Kernel Approximation", Submitted for publication(1999).

2. N. R. Aluru, "A Point Collocation Method Based On Reproducing Kernel Approximations", International Journal For Numerical Methods In Engineering, Vol. 47, pp. 1083 1121(2000).

3. N. R. Aluru, "A Reproducing Kernel Particle Method for meshless analysis of microelectromechanical systems", Computational Mechanics, Vol. 23, pp. 324-338(1999).

4. K. J. Bathe, E. Ramm, and E. L. Wilson, "Finite Element Formulations For Large Deformation Dynamic Analysis", International Journal For Numerical Methods In Engineering, Vol. 9, pp. 353-386(1975).

5. P. M. Osterberg and S. D. Senturia, "M-Test: A Test Chip for MEMS Material Property Measurement Using Electrostatically Actuated Test Structures", Journal of Microelectromechanical Systems, Vol. 6, No. 2, pp. 107 - 118(1997).

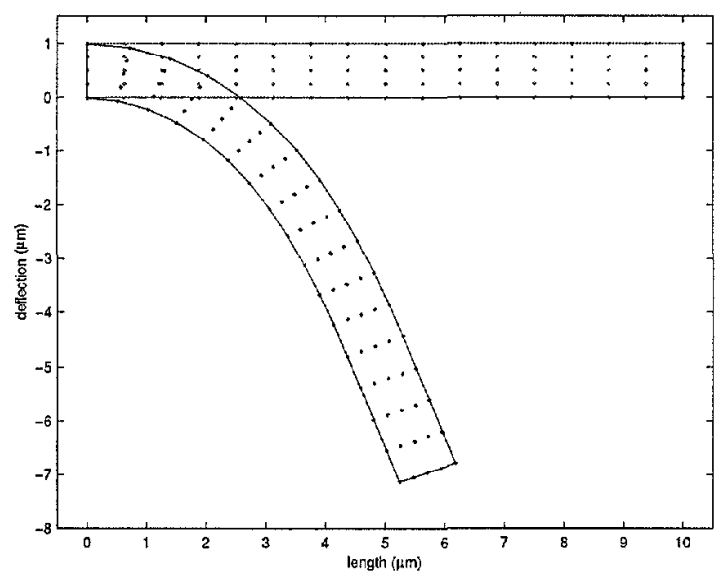

Figure 1. Deformed cantilever beam using a uniform point distribution.

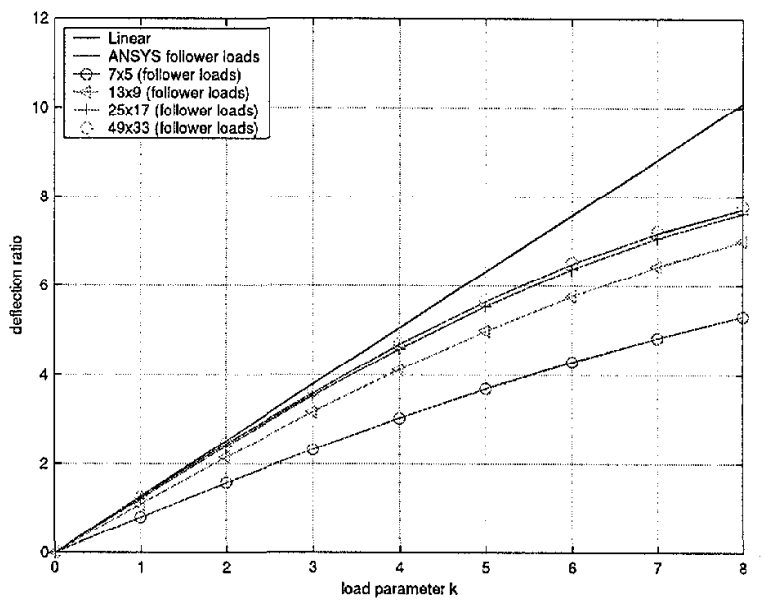

Figure 2. Point refinement to improve the accuracy of the solution.

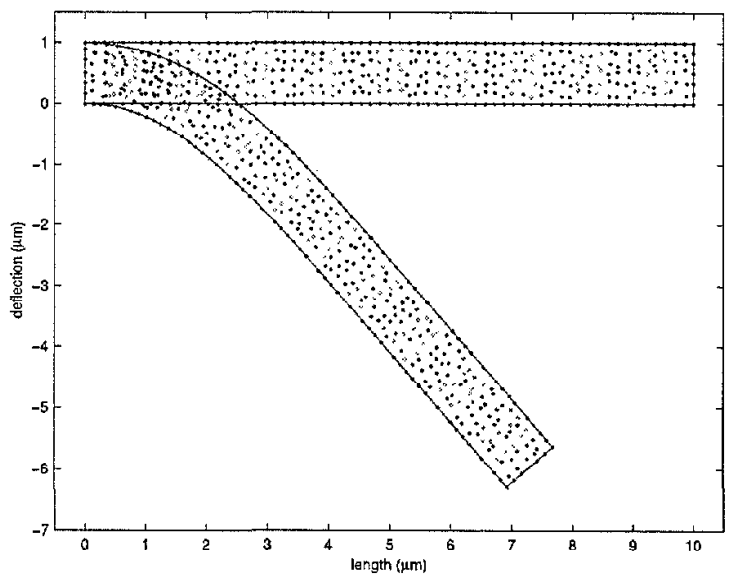

Figure 3. Deformed cantilever beam using a random distribution of points.

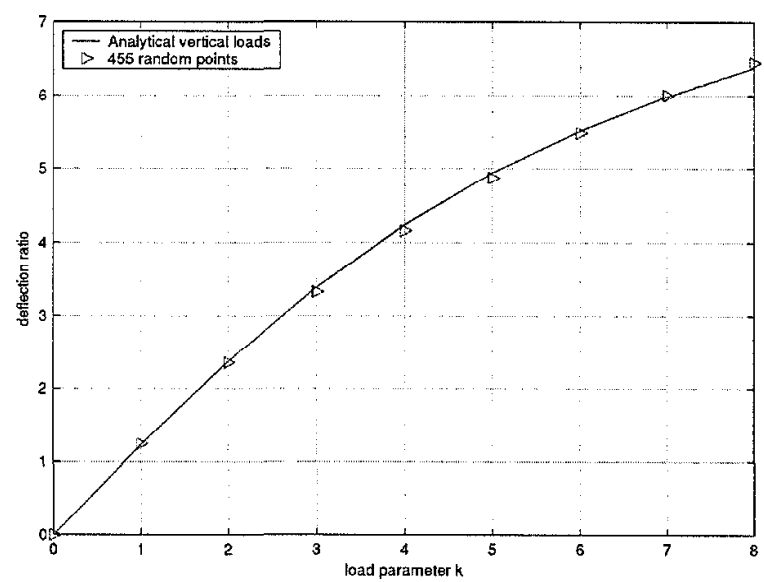

Figure 4. Comparison of the meshless solution (obtained with a random distribution of points) with the analytical solution. 


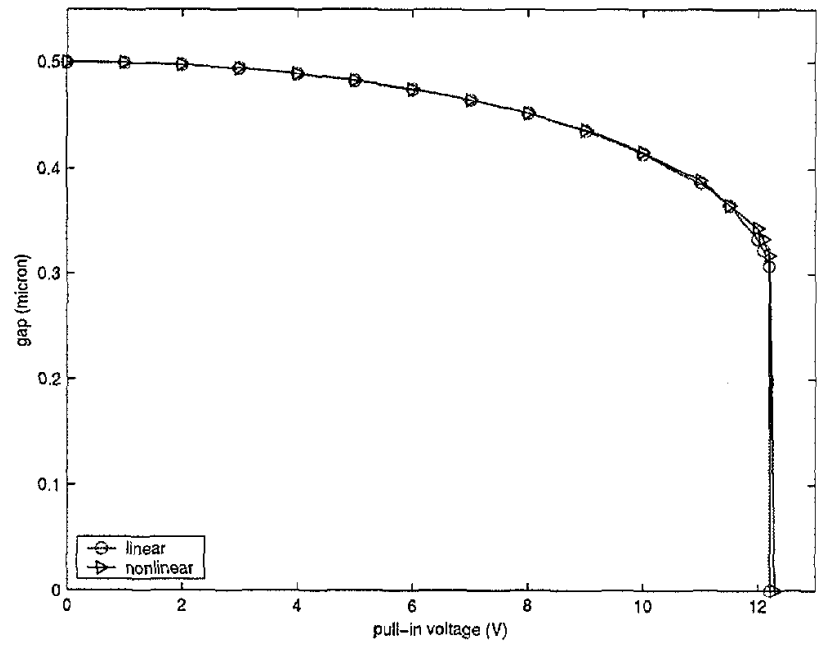

Figure 5. Comparison of Linear and non-linear results for a fixed-fixed beam with a length of $70 \mu \mathrm{m}$ and a gap of $0.5 \mathrm{um}$.

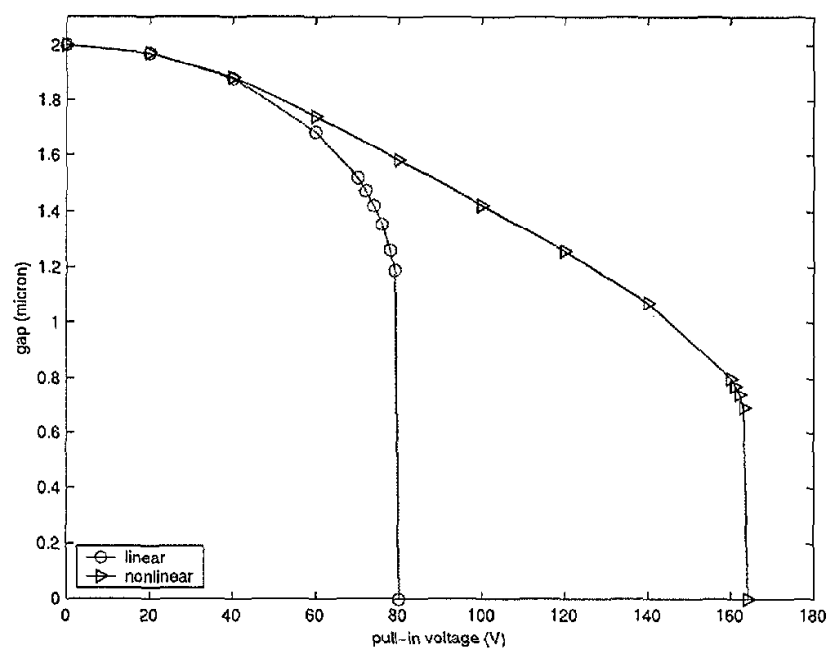

Figure 6. Comparison of Linear and non-linear results for a fixed-fixed beam with a length of $70 \mu \mathrm{m}$ and a gap of $2 \mu \mathrm{m}$.

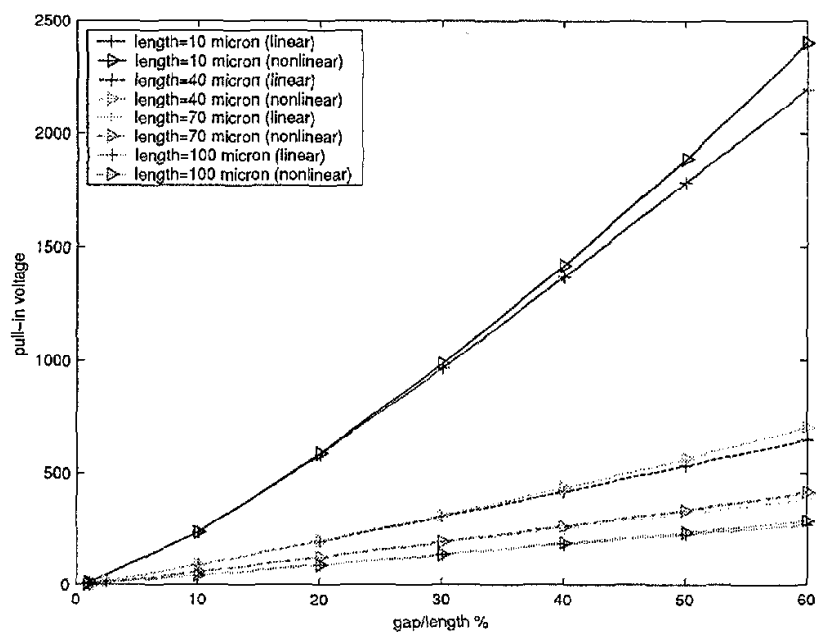

Figure 7. Gap/length ratio vs. pull-in voltage for cantilever beams.

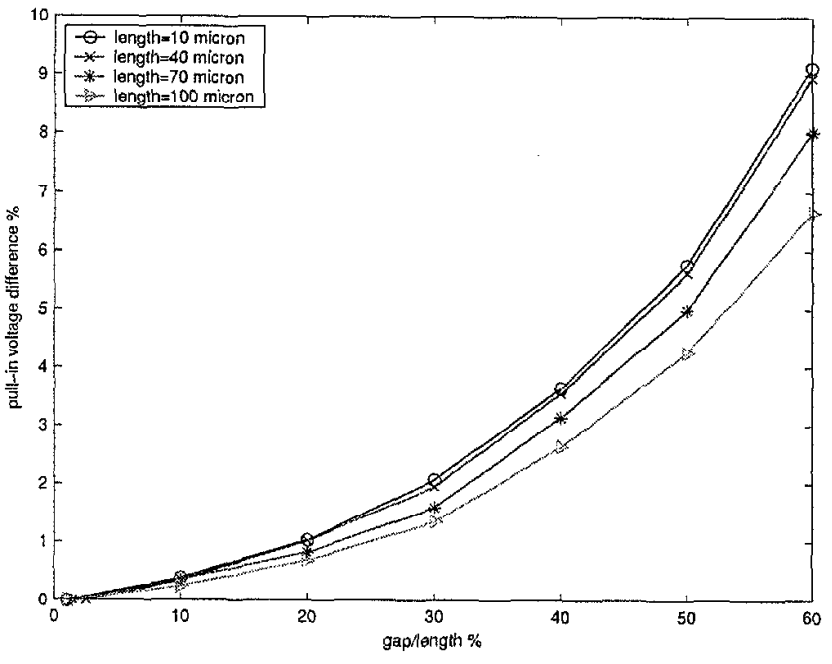

Figure 8. Gap/length ratio vs. pull-in voltage error between linear and non-linear results for cantilever beams.

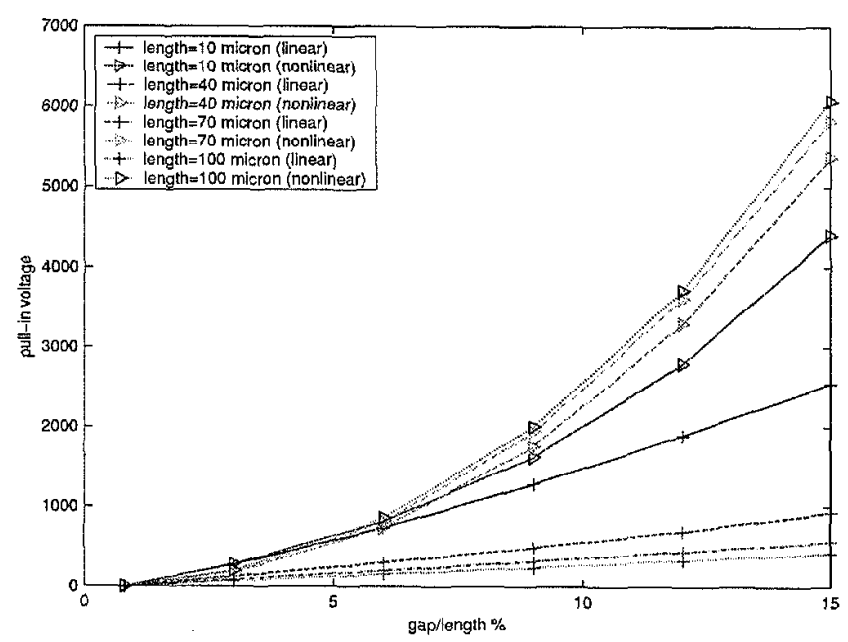

Figure 9. Gaphength ratio vs. pull-in voltage for fixed-fixed beams.

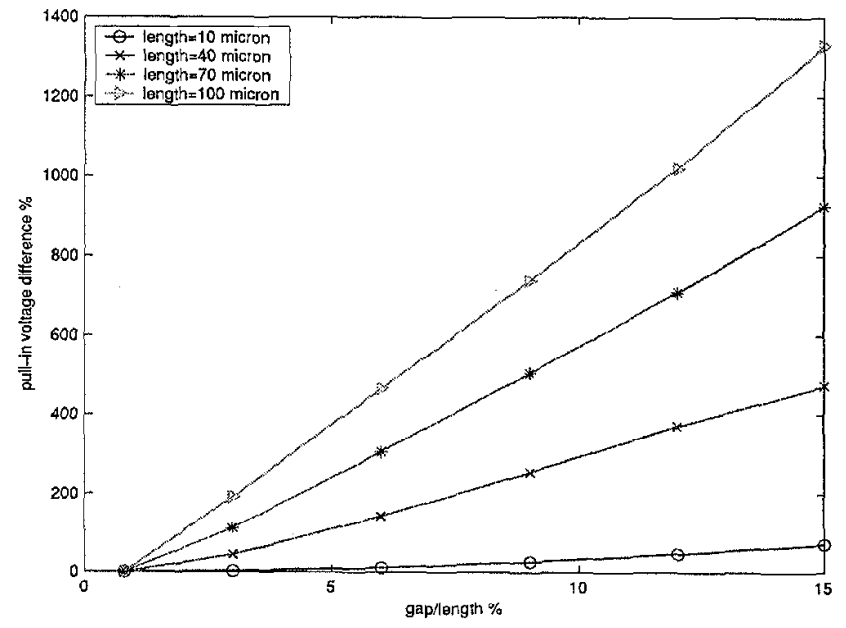

Figure 10. Gaphength ratio vs. pull-in voltage error between linear and non-linear results for fixed-fixed beams. 